\title{
HIGH SPEED UV PHOTOMETRY OF THE AM HER SYSTEMS AM HER, VV PUP AND V834 CEN, WITH THE HUBBLE SPACE TELESCOPE
}

\author{
K. SCHAEFER ${ }^{1}$, H. BOND ${ }^{1}$, G. CHANMUGAM ${ }^{2}$ \\ 1. STScI, 3700 San Martin Dr., Baltimore, MD 21218, USA \\ 2. Physics $\mathcal{E}$ Astronomy Dept., Louisiana State Univ., Baton \\ Rouge, LA 70803, USA
}

\begin{abstract}
We have used the High Speed Photometer (HSP) aboard the Hubble Space Telescope to observe the magnetic cataclysmic variables VV Pup, AM Her, and V834 Cen in the UV $(1400 \ldots 3300 \AA)$ with $0.01 \mathrm{~s}$ time resolution. We detected low frequency flickering in all three systems, and compare the time-scales with the predictions of King (1989). At higher frequencies we searched for shock oscillations from the accretion column(s) in these systems. The data were analyzed using the Gabor transform wavelet-like technique (Heil \& Walnut 1989) to search for frequency evolution throughout each observation. Preliminary analysis suggests the detection of rapid UV quasi-periodic oscillations (QPOs) in VV Pup at $0.74 \mathrm{~Hz}$, and at $4.4 \mathrm{~Hz}$ in V834 Cen. As in ground based observations, our observations failed to yield any rapid QPOs in AM Her itself.
\end{abstract}

\section{Introduction}

Theoretical studies of time dependent accretion onto highly magnetized white dwarfs predicted that the infalling gas forms a strong stand-off shock that is unstable for optically thin bremsstrahlung cooling (Langer, Chanmugam \& Shaviv 1981, 1982). The shock front was predicted to oscillate up and down above the white dwarf surface, with period:

$$
P \sim 1.1\left(\frac{A}{10^{16} \mathrm{~cm}^{2}}\right)\left(\frac{\dot{M}}{10^{16} \mathrm{~g} \mathrm{~s}^{-1}}\right)^{-1} R_{9}^{-1}\left(M / \mathrm{M}_{\odot}\right) \mathrm{s},
$$

where $\dot{M}$ is the accretion rate, $A$ is the cross sectional area of the accretion column, $M$ the white dwarf mass and $R_{9}$ the radius $R$ in units of $10^{9} \mathrm{~cm}$. 
These and other studies led to the discovery of $1 \ldots 2 \mathrm{~s}$ QPOs in VV Pup (Larsson 1989; Ramseyer et al. 1993) and V834 Cen (Larsson 1985; Middleditch et al. 1991). QPOs have not been detected from AM Her.

In addition to the rapid QPOs, large amplitude low frequency flickering has been seen in several AM Her binaries (see Cropper 1990). King (1989) has predicted that QPO (due to the motion and density of an ionization front near the $L_{1}$ point) in AM Her systems, with period:

$$
P \sim 5.510^{-2} P_{\text {orb }}
$$

may be seen. For AM Her, this corresponds to a period of $\sim 600 \mathrm{~s}$, and for VV Pup, $\sim 300$ s.

\section{Analysis and results}

Each $\sim 30 \mathrm{~m}$ observation was analyzed for both periodic and quasi-periodic signals in the frequency range $0<\nu$ (in $\mathrm{Hz}$ ) $<50$. Low amplitude QPO detections have been discovered at $\nu \sim 0.74,0.77 \mathrm{~Hz}$ for VV Pup (which was in a low state during the observations). VV Pup also exhibited long period flickering $(\sim 6 \mathrm{~m})$, similar to the prediction by King's model.

AM Her has not exhibited any rapid QPO, but did display large amplitude flickering with a period of $\sim 550 \mathrm{~s}$. This is again close to the period predicted by King (1989).

V834 Cen also showed weak, long period flickering, where $3.2<P$ (in $\mathrm{m})<8$. Analysis of the UV data has not yielded any QPOs stronger than $\sim 3 \sigma$, although analysis of the one $V$ band observation (also obtained with the HSP) has yielded an interesting, but low amplitude $4.4 \mathrm{~Hz}$ detection. Further investigation of this frequency and other phenomena seen in these data is underway.

\section{References}

Cropper, M., 1990, Space Sci. Rev., 54, 195

Heil, C.E., Walnut, D.F., 1989 SIAM Review, 31, 4, 628

King, A.R., 1989, MNRAS, 241, 365

Langer, S.H., Chanmugam, G., Shaviv, G., 1981, Ap. J., 311, L23

Langer, S.H., Chanmugam, G., Shaviv, G., 1982, Ap. J., 258, 289

Larsson, S., 1985, A\&A, 14, L1

Larsson, S., 1989, A\&A, 21, 146

Middleditch, J., Imamura, J.N., Wolff, M.T., Steiman-Cameron, T.Y., 1991, Ap. J., 382, 315

Ramseyer, T.F., Robinson, E.L., Zhang, E., et al., 1993, MNRAS, 260, 209 